\title{
Criminologie
}

\section{L’Association professionnelle des criminologues du Québec, dix ans après...}

Samir Rizkalla

Volume 10, numéro 2, 1977

La criminologie au Québec

URI : https://id.erudit.org/iderudit/017074ar

DOI : https://doi.org/10.7202/017074ar

Aller au sommaire du numéro

Éditeur(s)

Les Presses de l'Université de Montréal

ISSN

0316-0041 (imprimé)

1492-1367 (numérique)

Découvrir la revue

Citer cet article

Rizkalla, S. (1977). L’Association professionnelle des criminologues du Québec, dix ans après... Criminologie, 10(2), 62-63. https://doi.org/10.7202/017074ar d'utilisation que vous pouvez consulter en ligne.

https://apropos.erudit.org/fr/usagers/politique-dutilisation/ 


\section{L'ASSOCIATION PROFESSIONNELLE DES CRIMINOLOGUES DU QUÉBEC, DIX ANS APRES...}

Samir Rizkalla

Il y a exactement dix ans, en 1967, les premières démarches en vue de fonder l'Association professionnelle des criminologues du Québec étaient entamées. La charte fut signée officiellement le 28 août 1968.

A travers ces dix années, plusieurs conseils d'administration se sont succédé. On retrouve cependant de nombreux éléments de continuité notamment quant aux sujets de préoccupation et aux initiatives dont l'idée remonte aux premiers fondateurs de l'APCQ.

Lors d'une journée d'étuđes tenue le 28 février 1970 et à laquelle avaient participé 35 des 75 membres potentiels de l'Association, on discutait des points suivants : statut du criminologue ; information-innovation; relations avec le département de criminologie ; techniques d'intervention; conditions de travail...

Ces préoccupations demeurent les mêmes à quelques nuances près.

\section{Rencontres interprofessionnelles}

Par ailleurs, on retrouve dans les procès-verbaux de réunions qui remontent au début des années 1970 des textes qui parlent de création de services conjoints de secrétariat, ou autre, avec certaines associations.

Cette possibilité est encore envisagée à l'heure actuelle mais rien ne s'est matérialisé; d'une part, parce que l'APCQ a un pied-à-terre à l'École de criminologie, d'autre part, parce que les pourparlers interprofessionnels vont beaucoup plus loin que la simple mise en commun de services.

En effet, au cours des deux dernières années, les criminologues ont pris l'initiative de convoquer les exécutifs de diverses corporations et associations à des réunions, qui maintenant sont devenues régulières, et dont les objectifs sont les suivants : étudier les possibilités de regroupement ou de confédération en vue d'augmenter l'impact de nos professions respectives; prendre des positions conjointes dans les problèmes d'actualité pour lesquels nous avons un intérêt commun; examiner les questions d'ordre professionnel telles que l'évaluation, les codes d'éthique, les problèmes de recrutement, de cotisation, etc. ; enfin, envisager la possibilité de mettre en commun des services de soutien.

Les professionnels avec lesquels de telles négociations sont entreprises comprennent : les psychologues, les travailleurs sociaux, les conseillers en orientation, les psycho-éducateurs, les conseillers sociaux.

Il est important de mentionner deux problèmes majeurs autour desquels un travail conjoint a été amorcé ; il s'agit de c l'Éthique professionnelle et pratique syndicale , et du × projet de loi sur les jeunes délinquants $*$.

Il est certain que cette ligne de collaboration ne peut qu'être profitable à toutes les professions. Toutefois, nul n'a intérêt à perdre son identité de base d'où la lenteur et la prudence qui caractérisent les échanges actuels. 
Le criminologue, au milieu des professionnels si habilement appelés ARH (agents de relations humaines) par certains organismes du ministère des Affaires sociales, éprouve, nous semble-t-il, un double besoin, celui de s'affirmer comme tel, c'est-à-dire faire ressortir ce qui le distingue des autres, mais aussi celui de demeurer en étroite liaison avec eux pour avoir encore plus d'impact sur le plan professionnel et social.

Dix ans après, par conséquent, les nuances ont changé, mais la question fondamentale demeure la même !

Dans une étude faite lors de leur stage, deux étudiants de B.Sc. II, M. Lebeuf et D. Michelin (1976) écrivaient : ... il n'y a pas de praticien, comme tel, qui participe à ces diverses rencontres $»$. Ils parlaient ici des réunions tenues pour fonder l'APCQ.

Plus loin, ils soulignent : * Le 12 mars 1971, un nouveau conseil est élu... Tous les membres sont des praticiens excepté un directeur qui est professeur à l'École de criminologie.

On y lit enfin qu'en 1975 , on obtient un secrétariat à l'f́cole de criminologie, ainsi qu'un numéro de téléphones.

Ces trois citations refiètent un des aspects de la relation APCQ/Ecole, l'autre étant celui des échanges sur le plan de la formation et de la pédagogie.

Sur le plan de la relation stricte, on remarque donc que l'APCQ a été fondée au sein même de l'École, qui à l'époque était un département, par des étudiants ayant complété leur scolarité de maîtrise. Ce point de đépart créera à un moment donné, chez les praticiens, un désir de s'éloigner, de couper, en quelque sorte, le cordon ombilical. Ceci culmine en 1971 avec l'élection quasi exclusive de praticiens au Conseil d'administration.

Enfin, la relation se normalise et chaque entité cherche à établir un rapport égalitaire et d'intérêt commun avec l'autre. pédagogie.

Ceci est surtout perceptible au niveau de la formation et de la

Depuis le début des années 1970, on parle de \& rencontres de membres du conseil de l'APCQ avec le comité des professeurs $"$, on y discute de recherche, de stages, de formation des criminologues et de pratique de la criminologie $\gg$.

Le 21 mai 1976 une réunion rassemble praticiens et professeurs autour de la même table. Le premier contact est difficile et l'on peut même parler de confrontation. C'est normal, les praticiens en ont sur le cour de n'avoir pas été écoutés plus tôt, les professeurs se sentent un peu sur la défensive. Mais tout rentre rapidement dans l'ordre et, de cette première rencontre, naît l'idée de former un comité conjoint permanent APCQ/École.

Les mêmes problèmes évoqués timidement en 1970, avec force et presque agressivité en 1976, peuvent maintenant être abordés de façon sereine et pérenne.

Dix ans après, les criminologues rétablissent donc sur de nouvelles bases leurs rapports avec l'́cole de criminologie. 